\author{
MILITARY TEC:HNICAL COLLEGE \\ CAIRO - EGYPT
}

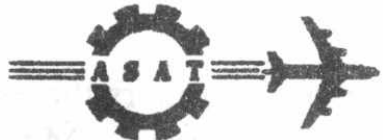

$7^{\text {th }}$ INTERNATIONAL CONF. ON AEROSPACE SCIENCES \& AVIATION TECHNOLOGY

\title{
Analysis of a 16-ary Quadrature Amplitude Modulation for Land Mobile Satellite Data Communication
}

\author{
Eweda Eweda, Salah El-Agooz, and Gamal Mabrouk \\ Military Technical College, Kobry EI - Koppa, Cairo, Egypt
}

\begin{abstract}
The paper is concerned with evaluating the performance of a 16-ary quadrature amplitude modulation (QAM) signaling when transmitted through a land mobile satellite channel. The channel exhibits fading and shadowing as well as additive white Gaussian noise. Fading and shadowing cause both envelope and phase variations of the received signal. The paper derives upper bounds of the probability of error due to each one of these variations. An expression of the irreducible probability of error is also derived. The derived expressions are useful for the design of land mobile satellite data communication systems.
\end{abstract}

\section{Introduction}

In a recent paper [1], we have evaiuated the probability of error of a quadraturepartial responce signaling when used in a shadowed rician fading land mobile satellite channel. The present paper extends the analysis given in [1] to the case of a 16-ary quadrature amplitude modulation (QAiV) signaling. Fig.1 shows a signal constellation of a 16-ary QAM system obtained from four levels on each quadrature channel. Fig.2 shows a basic QAM modulator and demodulator structure along with a representative waveform for 16-ary QAMI.

The performance of multilevel QAM operating in linear and nonlinear channels exhibiting Gaussian noise and other interference environments has been investigated by a number of researches[2-4]. However, much work is still required to investigate the performance of QAM in other important channels such as mobile satellite channel where fading and shadowing can affect the signaling performance. This paper provides an analysis of the probability of error of a 16-ary QAil signaling transmitted through a land 
mobile satellite channel. The paper considers the existence of both line-of sight (LOS) and multipath components of the received signal. Moreover, it considers shadowing by trees. The channel model used in the analysis is the one described in [5] and [6]. This model characterizes the combined effect of fading and shadowing of a land mobile satellite link in a rural environment. Due to shadowing, the LOS component follows a lognormal probability distribution. The multipath component is modeled as a narrow-band Gaussian random process whose envclope has a Rayleigh probability distribution. Analytical expressions for the probability of symbol error of a 16-ary QAM signaling due to envelope and phase variations caused by fading and shadowing will be given.

The paper is organized as follows: Section II is concerned with the analysis of error probability due to envelope variation. Aanalysis of error probability due to phase variations is given in section III. Section IV provides some numerical results. Section V concludes the paper.

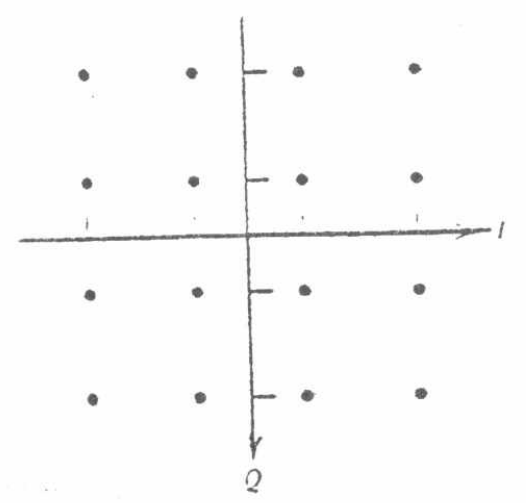

Fig. 1 Signal constellation of 16-ary QAM modulation

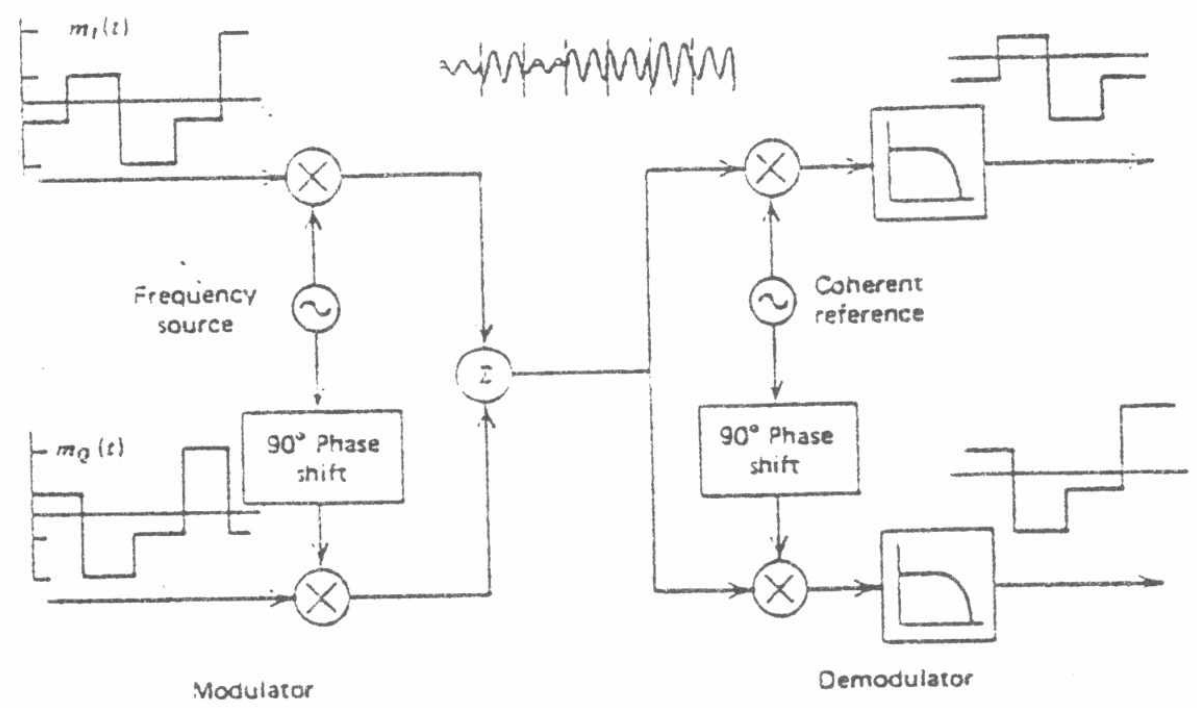




\section{U. Error Probability Due to Envelope Variation}

For an additive white Gaussian noise channel, the probability of error of al6-ary

QAMI signaling has the upper bound given by the following inequality[7]

$$
P_{c} \leq 4 Q\left(\frac{2 r}{\sqrt{5 N_{0}}}\right)
$$

Where $\mathbf{r}$ is the root mean square amplitude of the received signal, $\mathbf{N}_{0}$ is the noise power in bit-rate bandwidth, and the function $\mathrm{Q}(\mathrm{x})$ is defined as:

$Q(x)=\frac{1}{\sqrt{2 \pi}} \int_{x}^{\text {nn }} \exp \left(-\frac{u^{2}}{2}\right) d u$.

It can be shown that the two above equations yield

$$
P_{c} \leq 2 \exp \left(-\frac{2 r^{2}}{5 N_{0}}\right)
$$

In the case of a land - mobile satellite channel, $r$ is random due to random variations of the channel. The probability density of $r$ has been found in [5] and it is given by.

$$
P(r)=\frac{r}{\left(b_{0} \sqrt{2 \pi d_{0}}\right)} \int_{0}^{\infty} \frac{1}{z} \exp \left[-(\ln z-\mu)^{2} / 2 d_{0}-\left(r^{2}+z^{2}\right) / 2 b_{0}\right] \cdot I_{0}\left(r z / b_{0}\right) d z
$$

Where $I_{0}()$ is the modified Bessel function of zeroth order.

b. represents the average scattered power due to multipath,

$\begin{array}{cl}\mu & \text { is the mean value due to shadowing, and } \\ \sqrt{d_{o}} & \text { is the standard deviation due to shadowing. }\end{array}$

Now the probability of error due to the envelope variation can be obtained by averaging

(2) over $P(r)$. Thus,

$$
P_{0} \leq 2 \int_{0}^{\infty} P(r) \cdot \exp \left(-\frac{2 r^{2}}{5 N_{0}}\right) d r
$$

Substituting the expression of $P(r)$ of (3) into (4) and interchanging the order of integration gives

$$
P_{c} \leq \frac{2}{\sqrt{2 \pi d_{0}}} \int_{0}^{\infty} \int_{0}^{\infty} \frac{1}{z} \cdot \exp \left(-\frac{(\ln z-\mu)^{2}}{2 d_{0}}\right) \cdot \exp \left(-\frac{z^{2}}{2 b_{0}}\right) \cdot \frac{r}{b_{0}} \cdot \exp \left(-\frac{r^{2}}{2}\left(\frac{1}{b_{0}}+\frac{4}{5 N_{0}}\right)\right) \cdot I_{0}\left(\frac{r z}{b_{0}}\right) d r d z(5)
$$

Let:

$$
F_{1}=\int_{0}^{\infty} \frac{r}{b_{0}} \cdot \exp \left(-\frac{r^{2}}{2} a^{2}\right) \cdot I_{0}\left(\frac{r z}{b_{0}}\right) d r .
$$

$\mathrm{a}^{2}=\frac{1}{\mathrm{~b}_{0}}+\frac{4}{5 \mathrm{~N}_{0}}$

Using the substitution $\mathbf{u}=\mathbf{a r}$, then

$$
F_{1}=\frac{1}{a^{2} b_{0}} \int_{0}^{\infty} u \cdot \exp \left(-\frac{u^{2}}{2}\right) \cdot I_{0}\left(\frac{u z}{a b_{0}}\right) d u
$$


Let

$\gamma=\frac{z^{2}}{a^{2} b_{0}^{2}}$

Then, (7) implies that

$F_{1}=\frac{1}{a^{2} b_{u}} \cdot \exp \left(\frac{\gamma}{2}\right) \int_{n}^{\infty} u \cdot \exp \left(-\frac{u^{2}+\gamma}{2}\right) \cdot I_{o}(u \sqrt{\gamma}) d u$

The integrand in (9) is in the form of a Rice pdf [3]. Consequently,

$\int_{11}^{\infty} u \cdot \exp \left(-\frac{u^{2}+\gamma}{2}\right) \cdot I_{0}(u \sqrt{\gamma}) d u=1$

Then, (9) and (10) yield

$F_{1}=\frac{1}{a^{2} b_{0}} \cdot \exp \left(\frac{\gamma}{2}\right)$

After some algebraic manipulation, equations (5),(6) and (11) imply that, the average probability of error of a 16-ary QAM signaling due to the combined effect of shadowing and fading is bounded by

$P_{0} \leq \frac{1}{\sqrt{2 \pi d_{0}}}\left(\frac{10 N_{0}}{5 N_{0}+4 b_{0}}\right) \int_{0}^{\infty} \frac{1}{z} \cdot \operatorname{cxp}\left(-\frac{(\ln \%-\mu)^{2}}{2 d_{0}}\right) \cdot \operatorname{cxp}\left(-\frac{2 z^{2}}{\left(5 N_{0}++b_{0}\right)}\right) \cdot d z$

\section{Error Probability Due to Phase Variation}

The probability of error of a 16-ary QAM signaling with phase error is given by[7]

$\mathrm{P}_{\mathrm{e}} \leq 4 \mathrm{Q}\left(\frac{2 \mathrm{r}}{\sqrt{5 \mathrm{~N}_{\mathrm{o}}}} \cos \phi\right)$

where $\phi$ is the received signal phase error. Using the relation obtained by Jain and

$Q\left(\frac{r}{\sqrt{N_{0}}} \cos \phi\right)=\frac{1}{4}\left[1-\frac{2}{\pi} \sum_{n}^{n}(-1)^{n} \frac{\Gamma\left(n+\frac{1}{2}\right)\left(-\frac{r}{\sqrt{2 N_{0}}}\right)^{2 n+1}}{(2 n+1) !}, F_{1}\left(n+\frac{1}{2}, 2 n+2,-\frac{r^{2}}{2 N_{0}}\right) \cos (2 n+1) \phi\right](14)$

where,$F_{1}()$ is the confluent hypergeometric series defined in [11].

$\Gamma()$ is the gamma function.

In the case of fading and shadowing, $\phi$ is random. The probability density function of $\phi$ has been found to be approximately Gaussian [9]. Thus,

$$
P(\phi)=\frac{1}{\sqrt{2 \pi \sigma^{2}}} \exp \left(-\frac{(\phi-m)^{2}}{2 \sigma^{2}}\right)
$$

Where $m$ and $\sigma$ are the mean and the standard deviation of the signal phase,

respectively.

A performance estimate of a 16-ary QA.VI due to phase variation caused by fading and shadowing can be obtained by averaging (14) over $\mathbf{P}(\phi)$. Hence, the probability of error will be 
$P_{0} \leq 1-\left(\frac{2}{\pi} \sum_{0}^{\infty}(-1) \frac{\Gamma\left(n+\frac{1}{2}\right)\left(\frac{2 r}{\sqrt{10 N_{0}}}\right)^{2 n+1}}{(2 n+1) !} F_{1}\left(n+\frac{1}{2}, 2 n+2,-\frac{1}{\sqrt{5}} \frac{r^{2}}{N_{0}}\right) \cdot \int_{-\infty}^{\infty} \cos (2 n+1) \phi P(\phi) d \phi\right)(16)$

Rewrite the integration in (16) in complex form as:

$\int_{-\infty}^{\infty} \cos (2 n+1) \phi \mathbf{P}_{\phi}(\phi) d \phi=\operatorname{Re}\left[\int_{-\infty}^{\infty} \exp (j v \phi) \mathbf{P}(\phi) d \phi\right]$

where Re denote the real part and $v=2 n+1$.

The term between square brackets on the RHS of (17) is the characteristic function of $P(\phi)[11]$. Due to (15), one has

$\operatorname{Re}\left[\int_{-\infty}^{\infty} \exp (j v \phi) \frac{1}{\sqrt{2 \pi \sigma}} \exp \left(-\frac{(\phi-m)^{2}}{2 \sigma^{2}}\right) d \phi\right]=\operatorname{Re}\left[\exp \left(j v m-\frac{v^{2} \sigma^{2}}{2}\right)\right]$

Assuming that the phase has a zero mean, i.e. $\mathbf{m}=\mathbf{0}$, then $(16)-(18)$ yield

$P_{0} \leq 1-\left(\frac{2}{\pi} \sum_{0}^{\infty}(-1) \frac{\Gamma\left(n+\frac{1}{2}\right)\left(\frac{2 r}{\sqrt{10 N_{0}}}\right)^{2 n+1}}{(2 n+1) !} F_{1}\left(n+\frac{1}{2}, 2 n+2,-\frac{1}{\sqrt{5}} \frac{r^{2}}{N_{0}}\right) \cdot \exp \left(-\frac{(2 n+1)^{2}}{2} \sigma^{2}\right)\right.$

Equation (19) represents the probability of error for a 16-ary QAM signaling due to phase variation caused by fading and shadowing. For large, more than $15 \mathrm{~dB}$, values of signal-tonoise, one can approximate the confluent hypergeometric series by the following expression [10].

${ }_{1} F_{1}\left(n+\frac{1}{2}, 2 n+2,-\frac{1}{\sqrt{5}} \frac{r^{2}}{N_{0}}\right) \equiv \frac{\Gamma(2 n+2)}{\Gamma\left(n+\frac{3}{2}\right)}\left(\frac{1}{\sqrt{5}} \frac{r^{2}}{N_{0}}\right)^{-\left(n+\frac{1}{2}\right)}$

After some algebraic manipulation, (19) and (20) imply that

$P_{c} \leq 1-\frac{4}{\pi}\left(\sum_{n}^{n} \frac{(-1)^{n}}{(2 n+1)} \exp \left(-\frac{(2 n+1)^{2}}{2} \sigma^{2}\right)\right)$

Immediately, one notices that $(21)$ is independent of signal-to-noise ratio. This independence is due to the fact that for large signal-to-noise ratio, the effect of phase error dominates the effect of noise. Inquality (21) represents the irreducible probability of error of a 16-ary QAMI signal due to phase variation caused by fading and shadowing. This is the bottom effect of phase variation which cannot be down-crossed whatever large is the available SNR. Finally, it is worth mentioning that the effects of envelope variation and phase variation have been evaluated individually in this paper. The probability of symbol error due to the combined effect of envelope and phase variations is bounded from above by the sum of the individual error probabilities provided by (12) and (19).

\section{Numerical Results}

The results are obtained by numerically evaluating the probability of error expressions derived in the previous sections. Table I shows the channel model parameter examples used in the numerical calculation. Results for the error probability due to 
envelope variation are obtained using equation (12). The integral in (12) has been evaluated numerically using Gaussian quadrature integration.

Table I. Channel Model Parameters

\begin{tabular}{|c|c|c|c|}
\hline & $\begin{array}{l}\text { Wight shadowing and } \\
\text { fading }\end{array}$ & $\begin{array}{l}\text { Averase sliadowing } \\
\text { and fading. }\end{array}$ & $\begin{array}{l}\text { Heavy shadowing and } \\
\text { fading }\end{array}$ \\
\hline$b_{0}$ & 0.158 & 0.126 & 0.0631 \\
\hline$\mu$ & 0.115 & -0.115 & -3.91 \\
\hline$\sqrt{d_{0}}$ & 0.115 & 0.161 & 0.806 \\
\hline$\sigma$ & 0.36 & 0.45 & 0.52 \\
\hline
\end{tabular}

Fig. 3 shows the probability of error versus SNR for the cases of light, average, and heavy shadowing and fading. The resuits show that for the case of heavy shadowing and fading, a very high SNR is required to compensate for the effect of fading and shadowing. For example, at probability of $10^{-3}$, a margin of $34 \mathrm{~dB}$ is required. Also, for the same probability of error, margins of 14 and $19 \mathrm{~dB}$ are required to compensate for light and average shadowing and fading, respectively.

A 50-term approximation of the series in (1.9) was used to obtain numerical values of the probability of error due to phase variation. Also, a 1500-term approximation of the series in (21) was used to obtain results of the irreducible probability of error. The confluent hypergeometric series, ${ }_{1} F_{1}($.$) , was evaluated using a recurrence relation [10] and$ in terms of the modified Bessel functions of zero and first orders. As described by Jain and Blachman [9], in their evaluation of the confluent hypergeometric series for low probability of error or high SNR, terms not only need to be more accurate, but many terms are necessary for high SNR.

Fig.4 shows the probability of error versus SNR of a 16-ary QAM signal due to phase error caused by fading and shadowing. It was found that a 50-term approximation of (19) is adequate for the case of heavy shadowing and is reasonably accurate for the cases of light and average shadowing. This conclusion was crrived at by observing the differences in results between the irreducible probability of error calculation which is considered more accurate (with a 1500-term approximation) and the calculation of a 50term approximation.

Fig. 5 shows the probability of error of a 16-ary QAM signal at $\sigma$ ranging from $\sigma=$ 0.1 to 0.9 radian where $\sigma$ is the rms phase error. The results show, for practical systems, that the irreducible probability of error is very small when the rms phase error $\sigma$ is less than 0.3 radian. For larger values of $\sigma$, the irreducible probability of error increases rapidly as shown in Fig. 5

\section{Conclusions}

The symbol error probability of a 16-ary QAMI signaling has been evaluated for a land mobile satellite link in a rural environment. The presence of fading, shadowing, and additive noise has been considered. The analysis includes both envelope and phase variations caused by fading and shadowing. The irreducible error probability due to channel phase error has also been obtained. The results show that at a $10^{-3}$ probability of error, very high signal-to-noise ratio margins of 14,19 , and $34 \mathrm{~dB}$ are required to compensate for light, average, and heavy shadowing respectively when considering, envelope variation only. Asso, the results show that the irreducible probability of erroris 
less than $10^{3}$ when the rms phase error is less than 0.3 radian. For larger phase errors, the probability of symbol error increases rapidly and dominates the performance of the a 16-ary QAM.

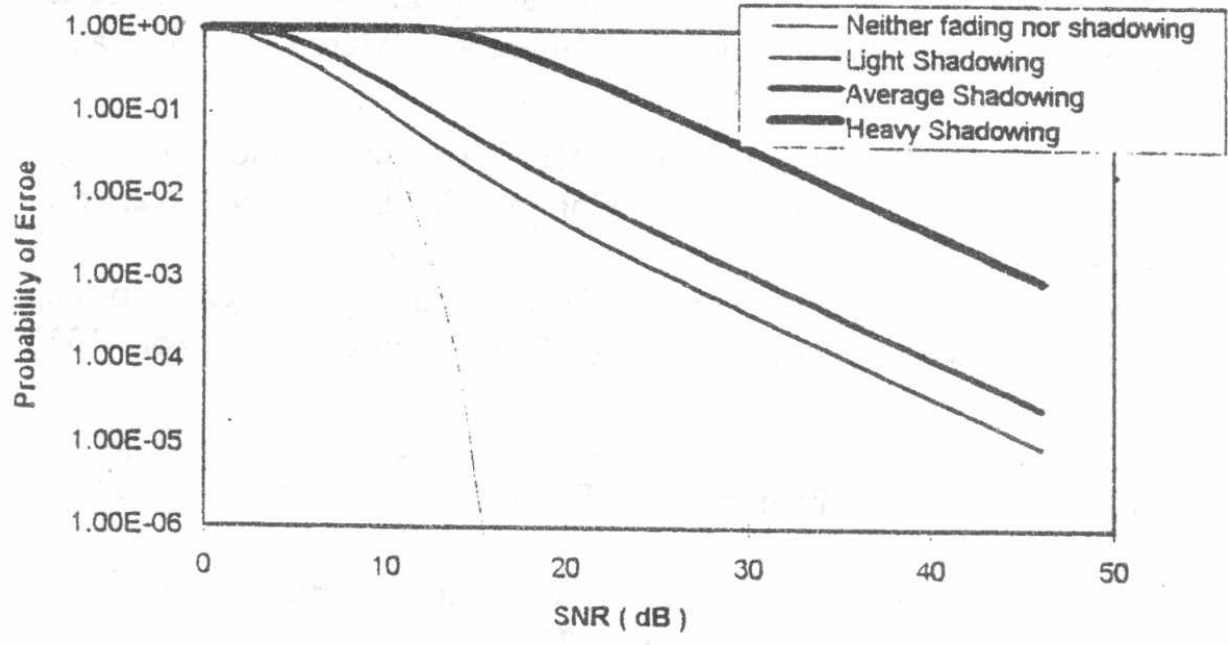

Fig.3 Probability of Error of a 16-ary QAM due to envelope variation.

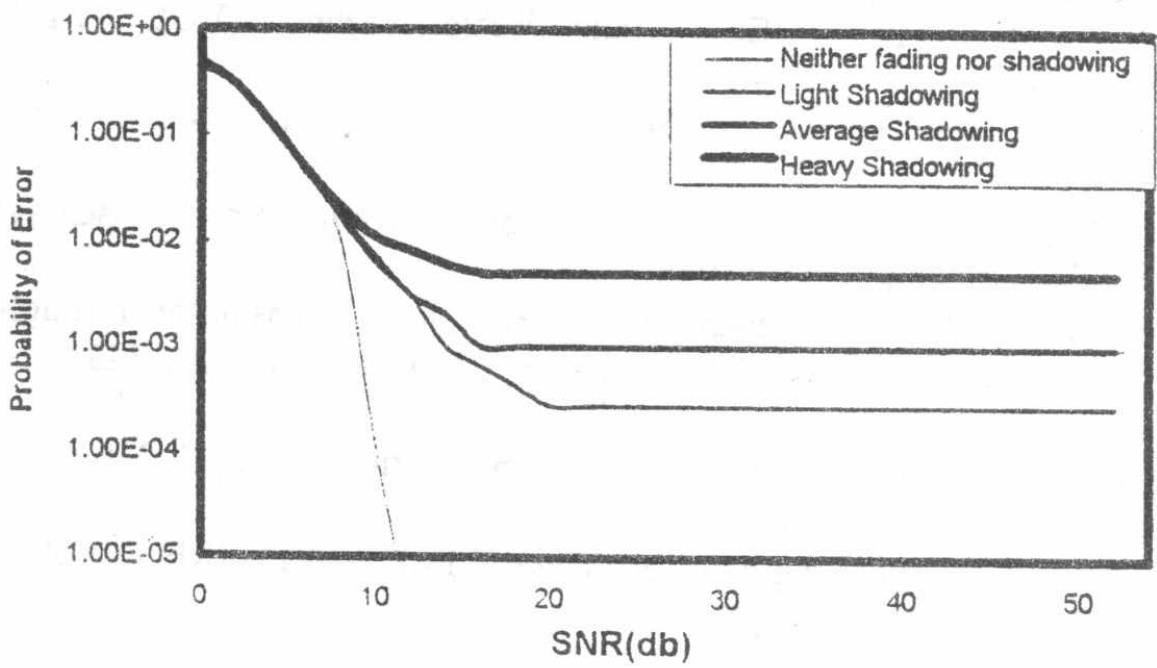

Fig.4 Probability of Error of a 16-ary QAM due to phase error caused by Light, Average, and Heavy Shadowing

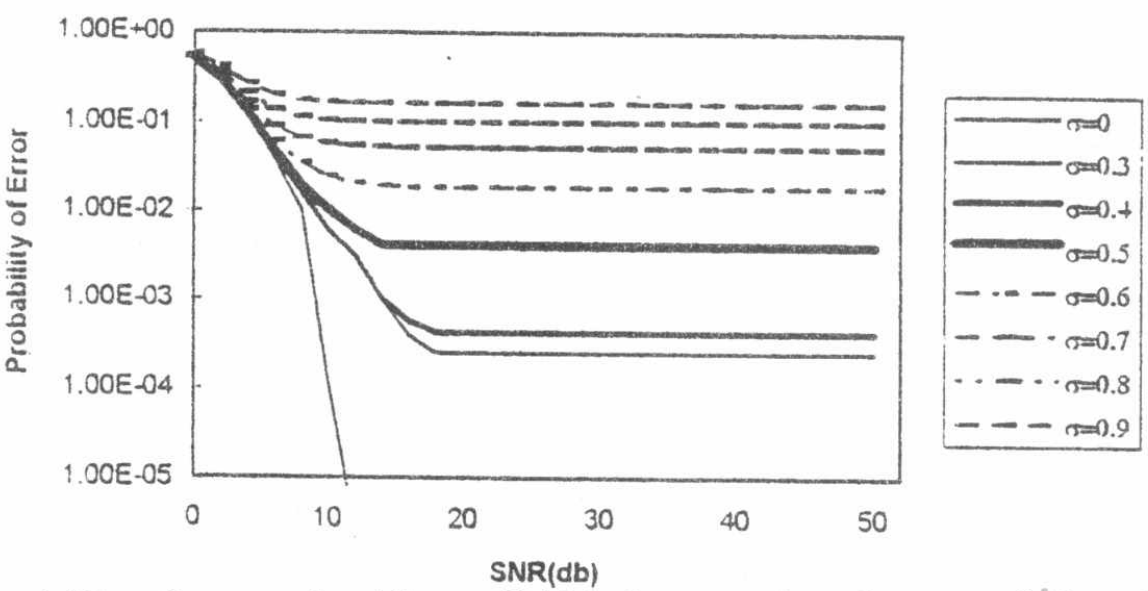

Fig.5 Probability of error of a 16-ary QAM at several variances of the phase error 


\section{References}

[1] E. Eweda, S. El-Agoez, and G. Mabrouk, "Performance Analysis of Quadrature Partial Responce Signaling in a shadowed Rician Fading Land Mobile Satellite Channel', Fourteenth National Radio Science Conference, March 23-25, Cairo university- Esypt, 1997.

[2] P.Dupuis, M.Joindat, A.Leclert and D.Soufflet, "16-QAM modulation for high capacity digital radio system", IEEE Trans. on Comm., VOL. 27, NO. 12, pp. 1771-1781, December 1979.

[3] A.H. Aghvami, "Performance analysis of 16-ary QAM signalling throught two-link nonlinear channels in additive Gaussian noise" , IEEE proc. on Comm., Vol. 131, No.4, pp. 403 - 406, July 1984.

[4] D.H.Morais and K. Feher , "NLA-QAM,A method for generating high power QAM signals throught nonlinear amlification”, IEEE Trans. on Comm., Vol. 30, No. 3, pp. 517 - 522, March 1982.

[5] C Loo, "A statistical model for a land mobile satellite link", IEEE Trans. Vehic Technology, Vol. VT - 34, pp. 122 - 127, Aug. 1985.

[6] C. Loo,"Measurments and Models of a land mobile satellite channel and their applications to MSK signals ", IEEE Trans. Vehic. Technol. ,VOL VT-36, pp.114-121, Aug. 1987.

[7] J.G.Proakis, Digital Communication, second eddition, McGraw - Hill, New York, 1989.

[8] S. O. Rice, "Statistical properties of a sine wave plus random noise ", Bell syst. Tech. J., Vol.27, pp. 109 - 117, Jan. 1948.

[9] P. C. Jain and N.M.Blachman, "Detection of a PSK signal transmitted through a hard-limited channel", IEEE Trans. Inform. Theory, VOL.IT - 19, pp. 623 - 630, Sept. 1973.

[10] D. Middleton, "An introduction to statistical communication Theory", New York: McGraw-Hill, pp. 1071-1076, 1960.

[11] W. R. Bennett, "Methods of solving noise problems," Proc. IEEE ( IRE ), Vol. 44 , pp. $609-638$, Nay 1956 